Case Report

\title{
A RARE CASE OF SCRUB TYPHUS MASQUERADING AS BREAST ABSCESS
}

\author{
Pothukuchi Venkata Krishna ${ }^{1}$, Venkata Ravikumar Chepuri', Sudhakar Kanumuri ${ }^{3}$ \\ Raghavareddy Yarram ${ }^{4} \&$ Sailaja Yadalla \\ ${ }^{1}$ Professor, ${ }^{2 \cdot 5}$ Postgraduates, Department of M edicine, Guntur M edical College Government General Hospital, \\ Guntur, Andhra Pradesh, India. \\ Correspondence : \\ Pothukuchi Venkata Krishna \\ 57-9-15, New Postal Colony, Patamata, Vijayawada - 520 010, Andhra Pradesh, India. \\ Mobile : +91 9848134088 E-mail : krishnapothukuchi@gmail.com
}

\begin{abstract}
:
We are presenting a case of scrub typhus masquerading as breast abscess in a pregnant woman who attended to hospital with history of fever and breast tenderness. A high index of suspicion is necessary as it is rarely seen but easily treatable and without proper and timely treatment, serious complications may arise which carries high mortality rate. Scrub typhus during pregnancy is quite rare. The line of treatment of scrub typhus in pregnancy is also analysed.
\end{abstract}

Keywords: Scrub typhus, eshcar, breast abscess

\section{Introduction:}

Scrub typhus is an acute febrile illness caused by Orientia tsutsugamushi (Rickettsia tsutsugamushi) which is principally a parasite of rodents and is transmitted by larval trombiculid mites (chiggers). ${ }^{[1]}$

In India, the presence of scrub typhus and other Rickettsial diseases has been known for several decades. During World War II, scrub typhus produced considerable morbidity and mortality among troops deployed In Southeast Asia. However, there has been a considerable decline in the incidence of scrub typhus in the later decades. Recent reports from several parts of India, including South India, indicate that there is a resurgence of scrub typhus. ${ }^{[2]}$ The clinical symptoms of scrub typhus in pregnant women are the same as in the non-pregnant. During pregnancy, scrub typhus may lead to spontaneous abortion, stillbirth, preterm delivery and small for

\section{Access this article online} Quick Response Code

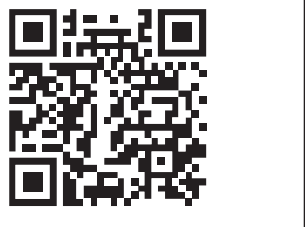
gestational age infants. ${ }^{[3]}$

Clinical picture of Scrub typhus is typically associated with fever, rash, myalgia and diffuse lymphadenopathy. A necrotic eshcar at the inoculating site of the mite is pathognomic of scrub typhus. Complications of scrub typhus usually develop after the first week of illness. Jaundice, renal failure, pneumonitis, ARDS, septic shock, myocarditis and meningoencephalitis are various complications known with this disease. ${ }^{[1]}$

For scrub typhus patients, rickettsial pox patients and some other rickettsiosis patients, an eschar 5-20 mm in diameter is formed at the area bitten by mites or ticks. The area bitten by trombiculid mites initially forms a papule, which becomes a vesicle and then an ulcer, and which is finally covered by a black eschar. The vicinity of the eschar is surrounded by red erythema, and an eschar is typically formed at the time of the manifestation of symptoms. ${ }^{[4,5]}$

\section{Case History :}

A 21year old female with 26weeks of pregnancy admitted in antenatal ward with history of fever of one week duration with painful swelling of right breast and body pains. She had received some antibiotic treatment outside. There was no history of jaundice, bleeding manifestations or other gastrointestinal disturbances. There was a history of right breast abscess one and a half year back which was drained surgically. Patient was a known case of asthma. There was no past history of diabetes or hypertension, nor any recent travel history. There was no history of trauma. 
On physical examination, patient was febrile, anemic, conscious and coherent. There was no jaundice and pedal edema. Patient was dyspneic with tachypnea, pulse rate was 106/min and blood pressure was 90/60 $\mathrm{mmHg}$. On examination bilateral occasional crepitations present in both lungs. Oxygen saturation was $80 \%$ at room air and 93\%with oxygen. Breast examination revealed tenderness in right breast with local rise of temperature along with a small ulcer along the medial aspect of the breast near the nipple and old healed surgical scar on the lateral aspect of the breast near the nipple area. Patient was treated for breast abscess with injection ceftriaxone and was referred to surgeon and physician. Surgeon advised continuation of same antibiotic treatment thinking of breast abscess. Physician advised bronchodilator inhalational therapy for bronchial asthma as oxygen saturation was low. Laboratory investigations revealed: Hemoglobin was 8.2 grams, total count was 7000 cell/cumm, platelet count was $92000 /$ cumm. Erythrocyte sedimentation rate was $20 \mathrm{~mm} / 1$ hour, Blood urea was $17 \mathrm{mg} / 100 \mathrm{ml}$, serum creatinine was $0.6 \mathrm{mg} / 100 \mathrm{ml}$, Serum sodium-153meq//tr, potassium-4.3meq/Itr, Chloride-118meq/Itr, bicarbonate$20 \mathrm{meq} / \mathrm{tr}$. Liver function tests were normal. Viral markers and strip test for malaria were negative. Ultrasound breast revealed subcutaneous edema surrounding the area of upper and outer quadrant, extending to the right axilla, suggestive of mastitis. Ultrasound abdomen showed mild hepatosplenomegaly, bilateral pleural effusion and a single live foetus of 27-28 weeks. Electrocardiogram showed tachycardia. Two Dimensional Echocardiogram showed features of hyperdynamic circulation with good ventricular function. Even after three days of antibiotic treatment fever was not subsided and her condition did not improve. The ceftriaxone was replaced with Injection augmentin and amikacin. But there was no improvement. Repeat 2D ECHO revealed minimal pericardial effusion. Again patient was referred to physician and transferred to acute medical care unit. On examination we noticed small eschar over an ulcerated area with purulent base on the medial aspect of breast (Figure-1). We suspected scrub typhus and oral Azithromycin 500mg per day was given and augmentin was continued. Blood sample was sent for Scrub typhus Elisa test for IgM and the report came as positive. Ulcer swab which was sent for gram stain and culture was negative. On second day of administration of azithromycin fever subsided and oxygen saturation was improved. Two days later, her condition improved and became normal.

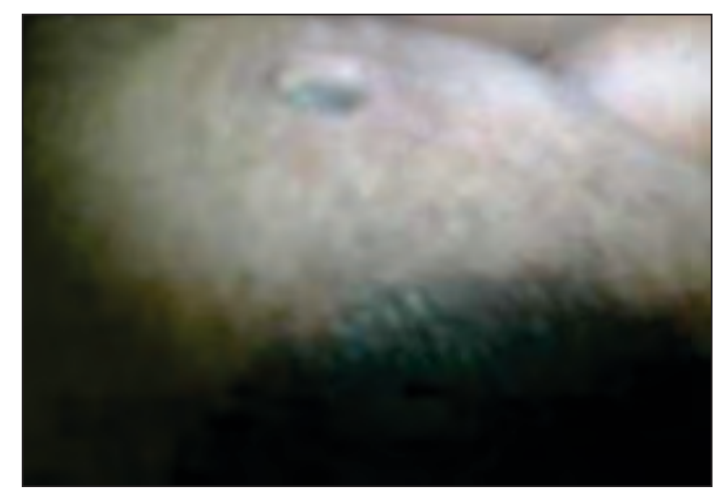

Figure1. : Eschar on breast

\section{Discussion :}

The eschar is the most useful diagnostic clue in patients with acute febrile illness in areas endemic for Scrub typhus and therefore should be thoroughly examined for its presence especially over the covered areas such as the groin, genitalia, infra-mammary area and axilla. ${ }^{[6]}$ The typical eschar is found to be a slightly raised erythema surrounding a black necrotic center. However, for cases in which an eschar is formed in a warm and damp area, i.e., the axilla area or the perineum, a necrotic eschar is not formed; instead, an ulcer with a shallow, purulent base surrounded by a clear, erythematous band may be formed; in such cases, the eschar could possibly be improperly diagnosed and may be easily overlooked. ${ }^{[5,7]}$

Though the eschar is painless, in this case because of soft tissue of breast and probably because of secondary infection of scrub typhus ulcer of breast, there was swelling and tenderness because of mastitis. Empiric treatment for 3 days with doxycycline, $100 \mathrm{mg}$ orally twice daily, or with minocyline, $100 \mathrm{mg}$ intravenously twice daily, or for 7 days with chloramphenicol, $25 \mathrm{mg} / \mathrm{kg} / \mathrm{d}$ orally or intravenously in four divided doses, eliminates most deaths and relapses. Chloramphenicol- and tetracycline-resistant strains have been reported from Southeast Asia, where azithromycin or 
roxythromycin may become the drug of choice for children, pregnant women, and patients with refractory disease. ${ }^{[1]}$ Chloramphenicol is classified as class $\mathrm{C}$ drug. Clinical data indicate that chloramphenicol is safe to use in pregnancy if it is not circulating at the time of delivery where it may cause gray baby syndrome. ${ }^{[8]}$ Ciprofloxacin, in experience with pregnant women in India, is ineffective and should not be used. ${ }^{[9]}$

We are reporting this case of Scrub typhus with breast abscess to highlight the need for strong suspicion especially in pregnant woman because of its dramatic response to treatment and keeping in mind of its severe life threatening complications.

\section{Conclusion :}

Scrub typhus is grossly under-diagnosed in India due to its

\section{References:}

1. Wayne X. Shandera, Ingrid I. Roig. Viral \& Rickettsial infections. In: Maxine A. Papadakis, Stephen J. McPhee, editors. Current Medical Diagnosis and Treatment, 52nded.USA: Mc Graw Hill Publishers;2013.p.1411-12

2. Vivekanandan M, M ani A, Priya YS, Singh AP, Jayakumar S, Purty S. Out Break of Scrub Typhus in Pondicherry. J Assoc Physician India 2010;58:24-28.

3. Vorapong Phupong. Pregnancy and Scrub typhus. J k Science 2010;12:85-7.

4. Berman SJ, Kundin WD. Scrub typhus in South Vietnam. A study of 87 cases. Ann Intern M ed 1973;79:26-30.

5. Dong-M in Kim, Kyung Jun Won, Chi Young Park, Ki Dong Yu, Hyong Sun Kim, Tae Young Yang, et al. Distribution of Eschars on the body of Scrub typhus patients: A Prospective Study. Am. J. Trop. Med. Hyg 2006;75:955-959.

6. Kundavaram A P, Jonathan A J, Nathaniel SD, Varghese G M . Eschar in scrubtyphus: A valuable clue to the diagnosis. J Postgrad Med 2013;59:177-8.

7. Irons EN, Armstrong HE. Scrub typhus in Dutch New Guinea. Ann Intern 1947;26:201-220.

8. Briggs GG, Freeman RK, Yaffe SJ. Drugs in pregnancy and lactation. 8th ed. Philadelphia: Lippincott Williams \& Wilkins; 2008.pp.345.

9. Mathai $E$, Rolain JM, Verghese $L$, et al. Case reports: scrub typhus during pregnancy in India. Trans R Soc Trop M ed Hyg 2003;97:570-72.

10. Chogle AR. Diagnosis and Treatment of Scrub Typhus -The Indian Scenario.J Assoc Physician India 2010;58:11-12. non specific clinical presentation limited awareness and low index of suspicion among clinicians, and lack of diagnostic facilities. ${ }^{[2]}$ Early diagnosis is important because there is usually an excellent response to treatment and timely anti-microbial therapy may help prevent complications. In developing countries with limited diagnostic facilities, it is prudent to recommend empiric therapy in patients with undifferentiated febrile illness having evidence of multiple system involvement. ${ }^{[10]}$ Scrub typhus should be listed in the differential diagnosis of acute febrile illness in pregnant women who either live in or return from endemic areas. The symptoms and signs during pregnancy are not different from non-pregnant women. Treatment with azithromycin is safe during pregnancy. ${ }^{[3]}$ 